\title{
The First Supernova Explosions in the Universe
}

\section{Citation}

Bromm, Volker, Naoki Yoshida, and Lars Hernquist. 2003. "The First Supernova Explosions in the Universe." The Astrophysical Journal 596 (2): L135-38. https://doi.org/10.1086/379359.

\section{Permanent link}

http://nrs.harvard.edu/urn-3:HUL.InstRepos:41381729

\section{Terms of Use}

This article was downloaded from Harvard University's DASH repository, and is made available under the terms and conditions applicable to Other Posted Material, as set forth at http:// nrs.harvard.edu/urn-3:HUL.InstRepos:dash.current.terms-of-use\#LAA

\section{Share Your Story}

The Harvard community has made this article openly available.

Please share how this access benefits you. Submit a story.

\section{Accessibility}




\title{
THE FIRST SUPERNOVA EXPLOSIONS IN THE UNIVERSE
}

\author{
Volker Bromm, NAOKI Yoshida ${ }^{1}$, ANd LARs Hernquist \\ Harvard-Smithsonian Center for Astrophysics, 60 Garden Street, Cambridge, MA 02138; \\ vbromm@cfa.harvard.edu,nyoshida@cfa.harvard.edu, lars@cfa.harvard.edu
}

\author{
Submitted to ApJ Letters
}

\begin{abstract}
We investigate the supernova explosions that end the lives of massive Population III stars in low-mass minihalos $\left(M \sim 10^{6} M_{\odot}\right)$ at redshifts $z \sim 20$. Employing the smoothed particle hydrodynamics method, we carry out numerical simulations in a cosmological set-up of pair-instability supernovae with explosion energies of $E_{\mathrm{SN}}=10^{51}$ and $10^{53} \mathrm{ergs}$. We find that the more energetic explosion leads to the complete disruption of the gas in the minihalo, whereas the lower explosion energy leaves much of the halo intact. The higher energy supernova expels $\gtrsim 90 \%$ of the stellar metals into a region $\sim 1 \mathrm{kpc}$ across over a timescale of $3-5$ Myr. Due to this burst-like initial star formation episode, a large fraction of the universe could have been endowed with a metallicity floor, $Z_{\min } \gtrsim 10^{-4} Z_{\odot}$, already at $z \gtrsim 15$.
\end{abstract}

Subject headings: cosmology: theory — galaxies: formation — hydrodynamics - intergalactic medium — stars: formation - supernovae: general

\section{INTRODUCTION}

One of the most important challenges in modern cosmology is to understand how the cosmic dark ages ended (e.g., Barkana \& Loeb 2001; Bromm \& Loeb 2003a). Recent numerical simulations have presented evidence that the first (Pop III) stars, formed out of metal-free gas in low-mass halos at redshifts $z \gtrsim 20$, were predominantly very massive with $M_{*} \gtrsim 100 M_{\odot}$ (Bromm, Coppi, \& Larson 1999, 2002; Abel, Bryan, \& Norman 2000, 2002). As the first stars mark the crucial transition from a smooth, homogeneous universe to an increasingly complex and structured one, the important question arises: How did the first stars die?

The answer to this question sensitively depends on the precise mass of a Pop III star. In particular, if the star has a mass in the narrow interval $140 \lesssim M_{*} \lesssim 260 M_{\odot}$, it will explode as a pair-instability supernova (PISN), leading to the complete disruption of the progenitor (Fryer, Woosley, \& Heger 2001; Heger et al. 2003). Pop III stars with masses below or above the PISN range are predicted to form black holes. This latter fate is not accompanied by a significant dispersal of heavy elements into the intergalactic medium (IGM), since most of the newly synthesized metals will be locked up in the black hole. The PISN, however, will contribute all its heavy element production to the surrounding gas.

How did the pristine IGM get enriched with the first heavy elements? To account for the widespread presence of metals in the Ly $\alpha$ forest at $z \sim 5$ (e.g., Songaila 2001), star formation in low-mass systems at $z \gtrsim 10$ has been proposed as a likely source (Madau, Ferrara, \& Rees 2001). The metals produced in these low-mass halos can more easily escape from their shallow potential wells than those at lower redshift (e.g., Aguirre et al. 2001a, b). In addition, the enriched gas has to travel much shorter distances between neighboring halos at these early times, and it might therefore have been easier to establish a uniform metal distribution in the IGM.

In this Letter, we present numerical simulations of the supernova explosions that end the lives of massive Pop III stars. In contrast to earlier work, both analytical (e.g., Larson 1974; Dekel \& Silk 1986; Scannapieco, Ferrrara, \& Madau 2002; Furlanetto \& Loeb 2003) and numerical (e.g., Mori, Ferrara, \& Madau 2002; Thacker, Scannapieco, \& Davis 2002; Wada \& Venkatesan 2003), we here focus on the minihalos that are the sites for the formation of the very first stars. The gas in these halos, with masses of $M \sim 10^{5}-10^{6} M_{\odot}$ and virializing at $z \gtrsim 20$, can only form stars in the presence of a sufficient amount of $\mathrm{H}_{2}$, the only viable coolant in these systems (e.g., Haiman, Thoul, \& Loeb 1996; Tegmark et al. 1997; Yoshida et al. 2003a).

An important motivation for this numerical study is to substantiate our earlier semi-analytical discussion of a fundamental transition in the character of star formation at high redshifts, from very massive Pop III stars to lowermass Pop II stars at $z \gtrsim 15$ (Mackey, Bromm, \& Hernquist 2003; see also Schneider et al. 2002). This transition redshift is an important ingredient in determining the reionization history of the universe, which has very recently been constrained by the WMAP satellite (e.g., Cen 2003; Ciardi, Ferrara, White 2003; Haiman \& Holder 2003; Wyithe \& Loeb 2003a,b; Sokasian et al. 2003; Yoshida et al. 2003b).

\section{NUMERICAL METHODOLOGY}

The evolution of the dark matter and gas components is calculated with the parallel version of GADGET (Springel, Yoshida, \& White 2001) in its "conservative entropy" formulation (Springel \& Hernquist 2002). This code combines the smoothed particle hydrodynamics $(\mathrm{SPH})$ method with a hierarchical (tree) gravity solver. Here, we briefly describe the additions to GADGET which are necessary for the investigation of explosions in zero-metallicity gas.

${ }^{1}$ National Astronomical Observatory of Japan, Mitaka, Tokyo 181-8588, Japan 
We include all the relevant cooling processes for gas temperatures $10^{2} \lesssim T \lesssim 10^{9} \mathrm{~K}$ (Cen 1992; Bromm et al. 2002), as well as heating due to the photoionization of $\mathrm{H}$, He, and $\mathrm{He}^{+}$. The chemical reaction network comprises the 9 species $\mathrm{H}, \mathrm{H}^{+}, \mathrm{H}^{-}, \mathrm{H}_{2}, \mathrm{H}_{2}^{+}, e^{-}, \mathrm{He}, \mathrm{He}^{+}$, and $\mathrm{He}^{++}$, including the reactions given in Haiman et al. (1996). Our implicit, backwards-differencing method to solve the coupled set of rate equations is fast and accurate (see Bromm et al. 2002).

To achieve the dynamical range required to follow the evolution of the gas from cosmological initial conditions down to the formation of high density clumps, which are the immediate progenitors of Pop III stars, and of the subseqent supernova (SN) blast waves, we have implemented a resampling technique within SPH (see Bromm \& Loeb $2003 \mathrm{~b}$ for details). This method of refining a coarser, parent simulation, and following the further collapse with increased resolution by 'splitting' the parent SPH particles into a number, $N_{\text {ref }}$, of child particles has already been successfully applied to problems in Galactic star formation (Kitsionas \& Whitworth 2002).

\section{SIMULATIONS: PRE-EXPLOSION PHASE}

\subsection{Initial Conditions and Clump Formation}

We initialize our simulations at $z_{i}=100$ according to a standard $\Lambda$ CDM model with parameters $\Omega_{m}=1-\Omega_{\Lambda}=$ $0.3, \Omega_{B}=0.045, h=0.7$, and $\sigma_{8}=0.9$, close to the values measured by WMAP (Spergel et al. 2003). Our computational box is periodic, has a comoving length of $100 h^{-1} \mathrm{kpc}$, and contains $128^{3}$ particles each in dark matter and gas. For the initial gas temperature we adopt the value of $200 \mathrm{~K}$. The fractional free-electron abundance is initialized as $x_{e}=4.6 \times 10^{-4}$, and the hydrogen molecule abundance as $f_{\mathrm{H}_{2}}=2 \times 10^{-6}$ (Anninos \& Norman 1996).

We follow the evolution until $z \simeq 20$, at which point the gas in one of the halos has collapsed to a density $n \gtrsim 10^{4} \mathrm{~cm}^{-3}$, thus violating the requirement that the local Jeans mass be resolved everywhere: $M_{J}>M_{\text {res }} \simeq 500 M_{\odot}$ (e.g., Bate, Bonnell, \& Bromm 2003). This first highdensity clump to form in the simulation comprises a mass of $\sim 10^{3} M_{\odot}$ within a radius of $\sim 1 \mathrm{pc}$. The clump lies at the center of a virialized dark matter minihalo of total mass $\sim 10^{6} M_{\odot}$ and virial radius $\sim 150$ pc. If allowed to collapse further, the gas in the clump would eventually form one very massive star (VMS), or a small multiple of them (see Abel et al. 2002; Bromm et al. 2002). Here, we assume that the clump will form a single VMS, producing ionizing radiation during its short lifetime before finally exploding as a PISN. Next, we describe the radiative feedback that precedes the explosion.

\subsection{Radiative Feedback}

To be able to simulate the propagation of the SN remnant into the surrounding gas with sufficient resolution, we have selected a Cartesian volume around the location of the high-density clump. The SPH particles within this volume of physical length $1 \mathrm{kpc}$ serve as the parent particles for the resampling procedure, such that each parent spawns $N_{\text {ref }}=16$ child particles (see Bromm \& Loeb $2003 \mathrm{~b}$ for details). After refinement, there are $\sim 5 \times 10^{5}$ SPH particles in the dark matter minihalo that hosts the PISN explosion, and the improved mass resolution is
$M_{\text {res }} \simeq 20 M_{\odot}$. The dark matter component, on the other hand, is not refined.

It is crucial to take into account the radiative feedback from the VMS on the density structure in the minihalo. Otherwise, we would severely overestimate the radiative losses in the early stages of the explosion. Recently, Bromm \& Loeb (2003c) have implemented an approximate scheme to address this radiative feedback that allows one to estimate the evolution of the central density field without carrying out a fully self-consistent radiative transfer calculation. We here briefly summarize this approach.

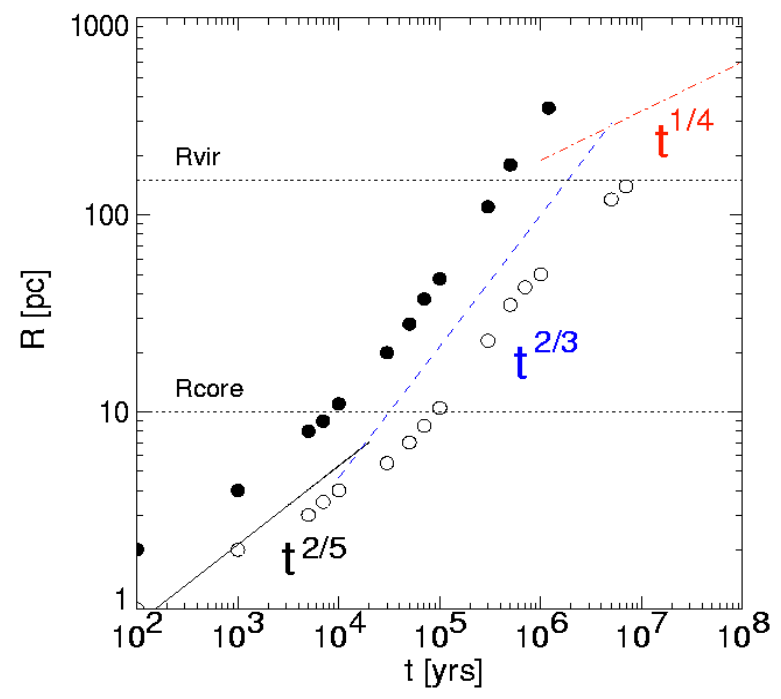

FIG. 1.- Evolution of the SN bubble. Shown is the radial position of the dense shell (in pc) vs. time after the explosion (in yr). Filled symbols: Simulation results for an explosion energy $E_{\mathrm{SN}}=10^{53}$ ergs. Open symbols: Simulation results for an explosion energy $E_{\mathrm{SN}}=10^{51} \mathrm{ergs}$. The lines show the analytical behavior of a SN blast wave. From early to late times: (i) Standard Sedov-Taylor solution. This is applicable within $r<R_{\text {core }}$ where the density is roughly constant. (ii) Sedov-Taylor evolution in a $\rho \propto r^{-2}$ density profile. This is the relevant case for $r>R_{\text {core. }}$ (iii) Finally, at times $t \gtrsim 10^{6} \mathrm{yr}$, radiative losses become important, and the SN remnant enters its snowplow phase.

We assume that the gas within the minihalo is rapidly ionized on a timescale that is short compared to the dynamical time in the halo. Specifically, we initialize our feedback simulation, aimed at determining the hydrodynamic response to the internal ionizing radiation, with a fully-developed H II region already in place. We choose a Strömgren radius of $R_{\mathrm{S}} \simeq 200 \mathrm{pc}$, and assume that the ionizing radiation is unattenuated within $R_{\mathrm{S}}$ (Osterbrock 1989). We now include photoheating terms in the entropy equation, such that (in units of ergs s $\mathrm{s}^{-1} \mathrm{~cm}^{-3}$ ): $\Gamma \propto r^{-2} \int \mathrm{d} \nu f_{\nu}\left(R_{*}\right) \sigma_{\nu}\left(\nu-\nu_{\text {th }}\right) / \nu_{\text {th }}$ for $r<R_{\mathrm{S}}$, and $\Gamma=0$ otherwise. Here, $f_{\nu}\left(R_{*}\right)$ is the flux at the surface of a very massive Pop III star (Bromm, Kudritzki, \& Loeb 2001b). We have also included the relevant photoreactions in the chemical reaction network (see Haiman et al. 1996). We allow the photoheating due to the central point source to go on for $\tau_{\mathrm{MS}} \simeq 2 \times 10^{6} \mathrm{yr}$, the approximate main-sequence lifetime of a VMS. Under the influence of the Pop III radiation field, the gas inside the $\mathrm{H}$ II region relaxes to a temperature of $T \simeq 3 \times 10^{4} \mathrm{~K}$, and the density within a central core of $R_{\text {core }} \sim 20$ pc reaches $n \sim 1 \mathrm{~cm}^{-3}$. 
At the end of the radiative feedback phase, we switch off the central point source, and initialize the SN explosion inside this photoheated density field. The detailed density structure very close to the VMS, at radii $r \lesssim 1 \mathrm{pc}$, is not reliably calculated by our approximate procedure. As we are here primarily interested in the evolution of the SN shell at radii $\gtrsim 10 \mathrm{pc}$, this caveat is not expected to compromise the main results in this study.

\section{SIMULATIONS: POST-EXPLOSION PHASE}

We carry out two explosion simulations. In each case, we assume that the Pop III star in the center of the minihalo explodes as a PISN. To span the possible energy range, we consider explosion energies of $E_{\mathrm{SN}}=10^{51}$ and $10^{53} \mathrm{ergs}$, corresponding to stars with masses $M_{*} \simeq 150$ and $250 M_{\odot}$ (Fryer et al. 2001). We focus in particular on the latter case, which marks the upper mass limit of the PISN regime. We insert the explosion energy as thermal energy, distributing it amongst $\sim 500 \mathrm{SPH}$ particles. These particles lie within an initial radius of $\sim 2 \mathrm{pc}$, such that the explosion is initialized with conditions appropriate for the adiabatic Sedov-Taylor (ST) phase. Since the PISN is completely disrupted without leaving a remnant behind, we can trace the subsequent fate of the metals by using the $\mathrm{SPH}$ particles that represent the stellar ejecta as markers.

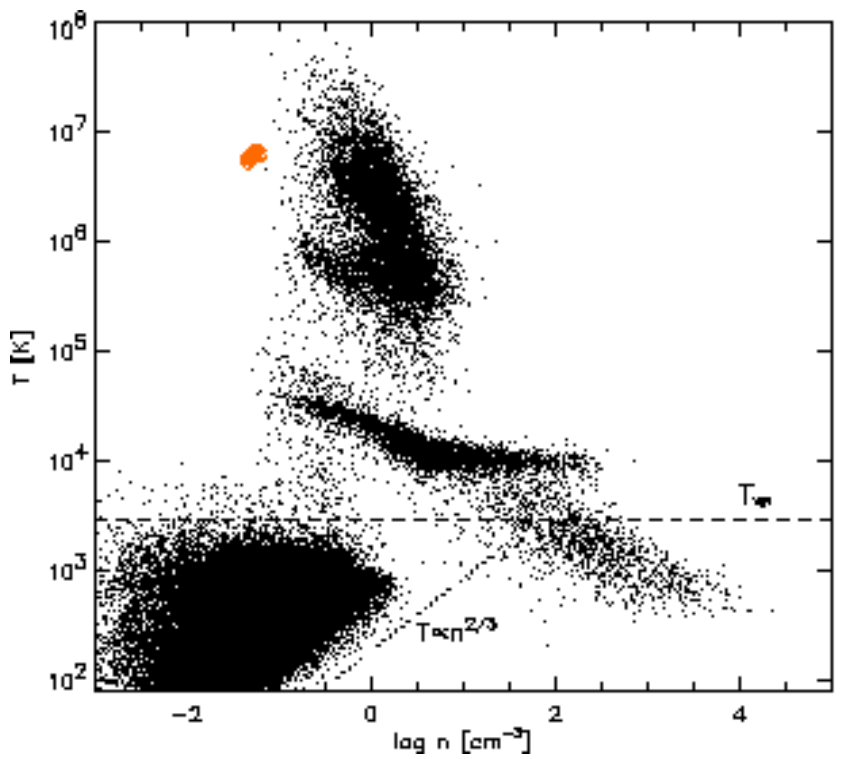

FIG. 2. - Thermodynamic properties of the shocked gas $\sim 10^{5} \mathrm{yr}$ after the explosion. Gas temperature (in K) vs. $\log n\left(\right.$ in $\left.\mathrm{cm}^{-3}\right)$. Dashed line: The virial temperature of the minihalo. It is evident that $T_{\mathrm{vir}} \sim 3000 \mathrm{~K}$ is much lower than the gas temperature in the minihalo. Most of the gas will therefore be expelled from the halo on a dynamical timescale. Dotted line: Adiabatic behavior. The gas in the outer reaches of the minihalo, as well as in the general IGM, approximately follows this scaling. Red symbols: Location of the gas that makes up the original stellar ejecta. This gas is hot and diffuse, can consequently cool only slowly, and therefore provides the pressure source that drives the expansion of the bubble. Notice that a fraction of the gas has been able to cool to $\sim 200 \mathrm{~K}$ due to the action of molecular hydrogen.

The expansion of the SN blast wave proceeds as shown in Figure 1, where we compare our simulations with simple analytical calculations. Initially, the blast wave evolves into a roughly uniform medium at radii $r \lesssim R_{\text {core }} \sim 20 \mathrm{pc}$, resulting in the usual ST scaling: $R_{\mathrm{sh}} \propto t^{0.4}$. Once the blast wave reaches beyond the core, however, it encounters an isothermal density profile in the remainder of the halo, leading to the scaling (e.g., Ostriker \& McKee 1988): $R_{\mathrm{sh}} \simeq 30 \mathrm{pc}\left(E_{\mathrm{SN}} / 10^{53} \mathrm{ergs}\right)^{1 / 3}\left(t / 10^{5} \mathrm{yr}\right)^{2 / 3}$. A few $10^{6} \mathrm{yr}$ after the explosion, radiative (inverse Compton) losses become important, and the SN remnant enters its final, snowplow phase. The significance of inverse Compton cooling distinguishes very high- $z$ SN explosions from those occuring in the present-day universe. From Fig. 1, it is evident that the numerical simulations nicely reproduce the analytical expectation.

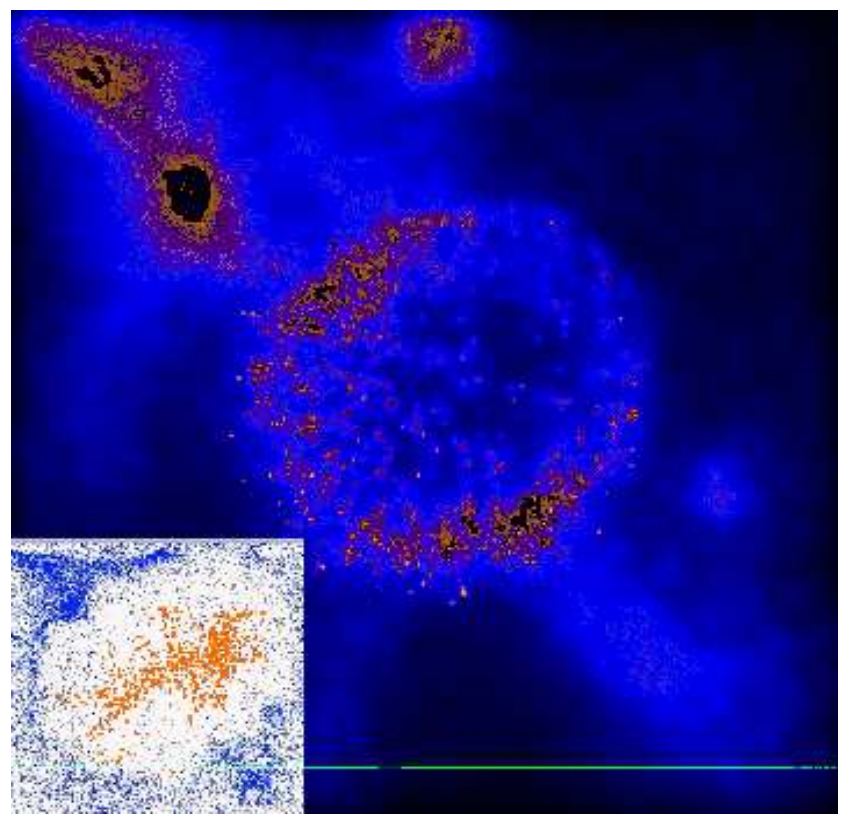

FIG. 3.- Situation $\sim 10^{6} \mathrm{yr}$ after the explosion for the case with $E_{\mathrm{SN}}=10^{53}$ ergs. Shown is the projected gas density within a box of linear physical size $1 \mathrm{kpc}$. The SN bubble has expanded to a radius of $\sim 200 \mathrm{pc}$, having evacuated most of the gas in the minihalo (with a virial radius of $\sim 150 \mathrm{pc}$ ). The dense shell has fragmented into numerous cloudlets. Inset: Metal distribution after 3 Myr. The stellar ejecta (red dots) trace the metals, and are embedded in pristine, un-enriched gas (blue dots). A large fraction $(\gtrsim 90 \%)$ of the heavy elements has escaped the minihalo, and filled a significant fraction of the hot bubble.

After approximately $10^{5} \mathrm{yr}$, when $t_{\text {cool }} \lesssim R_{\mathrm{sh}} / u_{\mathrm{sh}}$, a dense shell begins to form at a radius $\sim 50 \mathrm{pc}$. The thermodynamic properties of the dense shell, the interior bubble, and the surrounding medium are summarized in Figure 2. The shocked, swept-up gas exhibits three distinct thermal phases, as can clearly be discerned in Fig. 2: the first at $T \gtrsim 10^{5} \mathrm{~K}$, the second at $T \sim 10^{4} \mathrm{~K}$, and the third at $T \lesssim 10^{3} \mathrm{~K}$, with the latter two corresponding to the dense shell. The cooling from very high temperatures to $\sim 10^{4} \mathrm{~K}$, and subsequently to $10^{2} \mathrm{~K}$, proceeds in nonequilibrium, such that a fraction of free electrons persisits below $\sim 8,000 \mathrm{~K}$, allowing the re-formation of $\mathrm{H}_{2}$ (e.g., Shapiro \& Kang 1987; Oh \& Haiman 2002). The molecular fraction in the densest regions asymptotically assumes a 'plateau' value of $f_{\mathrm{H}_{2}} \sim 3 \times 10^{-3}$. Inside the expanding $\mathrm{SN}$ remnant lies a hot pressurized bubble (the red symbols in Fig. 2) that corresponds to the original stellar ejecta. This bubble drives the outward motion, and its gas is slowly cooled by adiabatic expansion and inverse Compton losses. Initially, cooling due to adiabatic expan- 
sion dominates (see the distribution of the red symbols in Fig. 2 as compared to the dotted line).

In Figure 3, we show the projected gas density $\sim 10^{6} \mathrm{yr}$ after the explosion for the $E_{\mathrm{SN}}=10^{53} \mathrm{ergs}$ case. Two features are apparent: Firstly, the blast wave has succeeded in completely disrupting the minihalo, dispersing most of the halo gas into the IGM. Comparing $E_{\mathrm{SN}}$ with the binding energy of the minihalo, $E_{\text {grav }} \sim 10^{51} \mathrm{ergs}$, such an outcome is clearly expected. It depends, however, on the inefficient radiative processes that cool the hot bubble gas in the initial stages of expansion. The evolution of the $E_{\mathrm{SN}}=10^{51} \mathrm{ergs}$ case indeed proceeds very differently. The blast wave effectively stalls at the virial radius, and the minihalo does not suffer complete disruption.

The second interesting feature in Fig. 3 is the vigorous fragmentation of the dense shell into a large number of small cloudlets. The emergence of these cloudlets, corresponding to gas at $T \lesssim 10^{3} \mathrm{~K}$, is not driven by gravity (e.g., Elmegreen 1994 and references therein), but is instead due to a thermal instability triggered by the onset of atomic cooling. The explosions simulated here do not result in the formation of lower-mass stars, or Pop II.5 stars in the terminology of Mackey et al. (2003). This is consistent with the prediction in Mackey et al. (2003) that Pop II.5 stars can only form in dark matter halos massive enough to be able to cool via atomic hydrogen (see also Salvaterra, Ferrara, \& Schneider 2003). To further test the possibility of SN triggered star formation in the high-redshift universe, we will explore a range of cosmological environments, corresponding to different halo masses and collapse redshifts, in future work.

What is the fate of the metals that are produced in the PISN progenitor? PISNe are predicted to have substantial metal yields, of the order of $y=M_{Z} / M_{*} \sim 0.5$, and for the largest stellar masses in the PISN range, most of $M_{Z}$ is made up of iron (Heger \& Woosley 2002). We find that in our $E_{\mathrm{SN}}=10^{53} \mathrm{ergs}$ simulation, $\sim 90 \%$ of the metals have escaped from the DM minihalo $4 \times 10^{6} \mathrm{yr}$ after the explosion. The metals have filled most of the interior hot bubble (see inset of Fig. 3), and there has not yet been sufficient time for them to significantly mix into the dense surrounding shell. The eventual metal distribution is rather flattened, with most metals ending up in the lowdensity voids. A rough estimate of the resulting metallicity in the surrounding, contaminated IGM can be obtained as follows: $Z=M_{Z} / M_{\text {gas }} \sim 100 M_{\odot} / 10^{5} M_{\odot} \gtrsim 10^{-2} Z_{\odot}$. This value is well above the critical metallicity threshold, $Z_{\text {crit }} \sim 10^{-3.5} Z_{\odot}$, such that very massive stars can only form out of gas with $Z<Z_{\text {crit }}$ (Omukai 2000; Bromm et al. 2001a; Schneider et al. 2002). We conclude that Pop III stars can continue to form in the pristine gas within the neighboring halos, as the metals that are dispersed in the explosion do not reach them.

\section{SUMMARY AND CONCLUSIONS}

We have simulated the death of the first stars in minihalos at $z \simeq 20$, assuming that they end their lives as PISNe. For the highest possible explosion energies, $E_{\mathrm{SN}}=10^{53} \mathrm{ergs}$, we find that a large fraction $(\sim 90 \%)$ of the stellar metals are dispersed into the surrounding IGM. The extent of the metal enriched region, with a physical size of $\sim 1 \mathrm{kpc}$, is comparable to the radius of the relic $\mathrm{H}$ II region around the minihalo. In a companion paper (Yoshida, Bromm, \& Hernquist 2003), we argue that if Pop III stars have led to an early partial reionization of the universe, as may be required by the recent WMAP results (e.g., Wyithe \& Loeb 2003b), this will have resulted in a nearly uniform enrichment of the universe to a level $Z_{\min } \gtrsim 10^{-4} Z_{\odot}$ already at $z \gtrsim 15$.

For the most energetic PISNe, these metals are predominantly in the form of iron, and the systems investigated in this Letter could have been responsible for an early burst of Fe enrichment. On nucleosynthetic grounds, such an initial episode of star formation has been inferred by Qian \& Wasserburg (2002). Indeed, the 'prompt inventory' postulated by these authors could have arisen in the PISN explosions studied here. Our simulations may also be relevant for explaining the recent observations of quasars at $z \gtrsim 6$, exhibiting surprisingly strong Fe II lines (Freudling, Corbin, \& Korista 2003). These authors have interpreted their results as hinting at an early nucleosynthetic contribution from Type Ia SNe, but it might be challenging to accomodate the required long evolutionary timescales, first to form a white dwarf, and then to sufficiently accrete from a binary companion to trigger the explosion. In fact, if our picture is correct that normal Pop II stars can only form at $z \lesssim 15$ (Mackey et al. 2003), the available time between $z \sim 15$ and $\sim 6$ is only $\sim 0.7$ Gyr, rendering the Type Ia scenario problematic. A PISN origin and the short timescales connected to it, however, could naturally account for the high $\mathrm{Fe} / \alpha$ ratios observed (see Heger \& Woosley 2002).

It would indeed be a remarkable feature of the universe, if the first stars had endowed the IGM with such a widespread, near-universal level of pre-enrichment.

We are grateful to Volker Springel for making available to us a version of GADGET. This work has been supported in part by NSF grant AST 00-71019. The simulations were performed at the Center for Parallel Astrophysical Computing at the Harvard-Smithsonian Center for Astrophysics.

\section{REFERENCES}

Abel, T., Bryan, G. L., \& Norman, M. L. 2000, ApJ, 540, 39

Abel, T., Bryan, G. L., \& Norman, M. L. 2002, Science, 295, 93

Aguirre, A., Hernquist, L., Schaye, J., Katz, N., Weinberg, D. H., \& Gardner, J. 2001a, ApJ, 561, 521

Aguirre, A., Hernquist, L., Schaye, J., Weinberg, D. H., Katz, N., \& Gardner, J. 2001b, ApJ, 560, 599

Anninos, P., \& Norman, M. L. 1996, ApJ, 460, 556

Barkana, R., \& Loeb, A. 2001, Physics Reports, 349, 125

Bate, M. R., Bonnell, I. A., \& Bromm, V. 2003, MNRAS, 339, 577

Bromm, V., Coppi, P. S., \& Larson, R. B. 1999, ApJ, 527, L5
Bromm, V., Ferrara , A., Coppi, P. S., \& Larson, R. B. 2001a, MNRAS, 328, 969

Bromm, V., Kudritzki, R. P., \& Loeb, A. 2001b, ApJ, 552, 464

Bromm, V., \& Loeb, A. 2003a, in AIP Conf. Proc. 666, The Emergence of Cosmic Structure, ed. S. S. Holt \& C. S. Reynolds (Woodbury: AIP), 73

Bromm, V., \& Loeb 2003b, ApJ, submitted (astro-ph/0212400)

Bromm, V., \& Loeb 2003c, ApJ, submitted

Cen, R. 1992, ApJS, 78, 341

Cen, R. 2003, ApJ, submitted (astro-ph/0210473) 
Ciardi, B., Ferrara, A., \& White, S. D. M. 2003, MNRAS, submitted (astro-ph/0302451)

Dekel, A., \& Silk, J. 1986, ApJ, 303, 39

Elmegreen, B. G. 1994, ApJ, 427, 384

Freudling, W., Corbin, M. R., \& Korista, K. T. 2003, ApJ, 587, L67

Fryer, C.L., Woosley, S. E., \& Heger, A. 2001, ApJ, 550, 372

Furlanetto, S. R., \& Loeb, A. 2003, ApJ, 588, 18

Haiman, Z., Thoul, A. A., \& Loeb, A. 1996, ApJ, 464, 523

Haiman, Z., \& Holder, G. P. 2003, ApJ, submitted (astro$\mathrm{ph} / 0302403)$

Heger, A., Fryer, C. L, Woosley, S. E., Langer, N., \& Hartmann, D. H. 2003, ApJ, submitted (astro-ph/0212469)

Heger, A., \& Woosley, S. E. 2002, ApJ, 567, 532

Kitsionas, S., \& Whitworth, A. P. 2002, MNRAS, 330, 129

Larson, R. B. 1974, MNRAS, 169, 229

Mackey, J., Bromm, V., \& Hernquist, L. 2003, ApJ, 586, 1

Madau, P., Ferrara, A., \& Rees, M. J. 2001, ApJ, 555, 92

Mori, M., Ferrara, A., \& Madau, P. 2002, ApJ, 571, 40

Oh, S. P., \& Haiman, Z. 2002, ApJ, 569, 558

Omukai, K. 2000, ApJ, 534, 809

Osterbrock, D. E 1989, Astrophysics of Gaseous Nebulae and Active Galactive Nuclei (Mill Valley: University Science Books)

Ostriker, J. P., \& McKee, C. F. 1988, Rev. Mod. Phys., 60, 1

Qian, Y.-Z., \& Wasserburg, G. J. 2002, ApJ, 567, 515
Salvaterra, R., Ferrara, A., \& Schneider, R. 2003, MNRAS, submitted (astro-ph/0304074)

Scannapieco, E., Ferrara, A., \& Madau, P. 2002, ApJ, 574, 590

Schneider, R., Ferrara, A., Natarajan, P., \& Omukai, K. 2002, ApJ, 571,30

Shapiro, P. R., \& Kang, H. 1987, ApJ, 318, 32

Sokasian, A., Abel, T., Hernquist, L., \& Springel, V. 2003, ApJ, submitted (astro-ph/0303098)

Songaila, A. 2001, ApJ, 561, L153 (erratum 568, L139 [2002])

Spergel, D. N., et al. 2003, ApJ, submitted (astro-ph/0302209)

Springel, V., Yoshida, N., \& White, S. D. M. 2001, NewA, 6, 79

Springel, V., \& Hernquist, L. 2002, MNRAS, 333, 649

Tegmark, M., Silk, J., Rees, M. J., Blanchard, A., Abel, T., \& Palla, F. 1997, ApJ, 474, 1

Thacker, R.J., Scannapieco, E., \& Davis, M. 2002, ApJ, 581, 836

Wada, K., \& Venkatesan, A. 2003, ApJ, in press (astro-ph/0303449)

Yoshida, N., Abel, T., Hernquist, L., \& Sugiyama, N. 2003a, ApJ, in press (astro-ph/0301645)

Yoshida, N., Bromm, V., \& Hernquist, L. 2003, ApJ, submitted

Yoshida, N., Sokasian, A., Hernquist, L., \& Springel, V. 2003b, ApJ, submitted (astro-ph/0303622)

Wyithe, S. B., \& Loeb, A. 2003a, ApJ, 586, 693

Wyithe, S. B., \& Loeb, A. 2003b, ApJ, 588, L69 